\title{
Imprints of COVID-19 Pandemic on the English Language: A Multidimensional Understanding
}

\author{
${ }^{* 1} \mathrm{Mr}$ Parveen Kumar and 2Dr Shachi Sood \\ ${ }^{1} M A$, MPhil, Department of English, Baba Ghulam Shah Badshah University, Rajouri, J\&K- 185132 \\ 2 Assistant Professor, Department of English, Baba Ghulam Shah Badshah University, Rajouri, J\& K- 185132
}

\begin{abstract}
The importance of English Language on the global level is undeniable and has been agreed upon in an array of discourses over this subject. This has also made it pertinent to understand how the English language has been affected by and responded to the COVID-19 pandemic that has touched almost every aspect of human life-Language being amongst the most affected. The present study has aimed to understand the multiple imprints of the pandemic over the English language and seeks to comprehend the ways in which it has posed challenges in front of the academia to understand the relevance of the language more profoundly during the time of crises. The paper has attempted to examine the evolving of English as a language during the pandemic and locates the areas in which the imprints necessitate further analysis.
\end{abstract}

Keywords: Communications, Language, Learning, Metaphors, Pandemic, Stories, Virtual

\section{Article Publication}

眮 Published Online: 20-Jan-2022

*Author's Correspondence

8 Mr Parveen Kumar

8 MA, MPhil, Department of English, Baba Ghulam Shah Badshah University, Rajouri, J\&K- 185132

\section{$\underline{10.31305 / \text { rrijm.2022.v07.i01.001 }}$}

$\triangle$ parveenkumar78089[at]gmail.com

C 2022 The Authors. Published by RESEARCH REVIEW International Journal of Multidisciplinary. This is an open access article under the CC BY-

NC-ND license

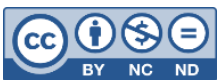

(https://creativecommons.org/licenses/ by-nc-nd/4.0/)

\section{Introduction}

It is an unquestionable fact that the pandemic COVID-19 has casted myriad effects over the multiple dimensions of human life. Whereas it is generally visible in the social, medical, and economic spheres, its effect has also shaken the very essence of language that constitutes our world. Even if we navigate through history, we can learn that major events such as wars and natural disasters affect the language on a larger scale. The numerous changes during the time of pandemic have led to many new ways of communication as well and changed the way in which the language has been used in the pre-pandemic era.

Therefore, it is an interesting endeavour to understand how the pandemic has intersected particularly through the English language in many ways and to search answers for the new questions that make us reconsider the ways in which the language evolves with time. It has also made us evaluate the outcomes of such intersections and consider the role of language in sharing the information during the pandemic and the changes in everyday language that arise due to the unpredictable disasters such as the prevalent pandemic. To have such a holistic understanding necessitates a comprehensive analysis of the imprints that the pandemic has casted upon the English language and the present study is an attempt towards the fulfillment of this endeavour.

\section{Pandemic and Translation}

Translation and communication are mostly interdependent upon each other because the primary function of translation is to communicate the knowledge from one language to the other. Since the pandemic hit the globe, a 
major challenge in front of the whole health care system has been the dissemination of pandemic related information equally to every corner of the world- in which there is a great linguistic diversity. To spread certain and reliable knowledge about the virus has been an indispensable part of communicating the virus-related information to the world. As in English Literature, translation helps a literary work to reach a global audience, similarly during the times of the pandemic, the translation has made it easier for medical organizations to take a better control over the situation and to maximize the COVID appropriate behaviour across many regions. Without translation it would not have been easy to disseminate reliable information across the globe. Translation has also facilitated the worldwide treatment rates because of the high and accurate tendency of the languages in which the treatment plans were circulated in a shorter span of time. It would not be an exaggeration to point out that in the time of COVID-19 crisis, translation has proved its significance at the global level.

\section{Disruption and Substitution of Non-verbal Communication during the Pandemic}

Language is not only about the written and spoken words and it almost seems lacking without the non-verbal gestures and signs that are deeply rooted in the spoken communication. However, the pandemic has at large changed the ways in which the non-verbal communication was prevalent in the cultures prior to the pandemic. The crisis has given rise to many new forms of non-verbal communication signs that act as a substitution for the greetings, gestures and other non-verbal cues that were dominant in the English language. A number of new forms of non-verbal communication techniques such as elbow bumps, fist bumps, squinting the eyes, arched eyebrows, tilted head etc.

It has been widely accepted that protective measures such as physical distancing and face masks that are taken against the pandemic have largely affected interpersonal communication. A decrease in the non-verbal communication signs can often lead to lack of comprehension and misunderstanding as well. In this connection, the use of face masks has led to recognize and utilise the significance of upper face through the eye-brows, eyes and upper cheeks during interpersonal communication and also to compensate the use of face by emphasizing the use of other body parts and using the body language simultaneously with the spoken language.

\section{A Rise in Neologisms}

Pandemic has affected the English language in many ways and one of the most visible aspects of it is the way in which it has introduced a variety of new words and coinages that were behind the curtains in the English language earlier. It is further an interesting endeavour to investigate the established trend and the nature of the neologisms that have emerged during the pandemic crisis. This can also be taken as a demonstration of the vitality and flexibility of the English language that can respond to any emerging situation.

Moreover, these neologisms have entered into many other languages and have been a component of daily use in most of the situations. The linguistic innovations and coinages that have been made in the English language during the pandemic include word-blending, acronyms, word-formations, compounding, and affixations among others. Some of the most trending neologisms that have emerged during the COVID-19 pandemic are the formations such as WFH (Work From Home), elbow-bump, smizing (smiling with the eyes while wearing a mask), mask down (a disregarded lockdown), Homeference ( a conference scheduled from the home), Qurantrolls (trolls made over the quarantine), covidiot (a person who foolishly disobeys the Covid protocols), Morona (a funny and derogatory term for Corona), Quaranteens (the teens growing during the time of Quarantine), Coronashaming, Quarantimes, Coro Apocalypse, Covideo party, and Coronaviva (a viva taken during the corona times) etc. These neologisms have made us understand and accept that language changes along with the global societal changes and English has entered into the very texture of most of the languages that are spoken across the world.

\section{Pandemic-related Imagery and Metaphors}

Metaphors have been used variously to make the people aware of the pandemic while also making it easy for them to relate it with the knowledge that they already possess. It is also an addition to the English language as we can 
analyse many metaphors and images that have evolved during the pandemic and thus became specific aspects of pandemic related communication. These metaphors include war metaphors; such as those that refer to the pandemic as an enemy, a traitor, a terrorist, an invader, and other alternative metaphors such as fire and sea metaphors and death imagery.

The words that have been metaphorically used in the English language to refer to different aspects of the pandemic include 'enemy', 'traitor', 'alien invader', 'corona fighters', and 'warriors'. This has made the virus seem as a malevolent opponent and an enemy that must be defeated with all the force. The military metaphors that have been widely used to refer to pandemic have in turn made it cause more anxiety and terror regarding the virus among the people. The emerging metaphors like these necessitate a reconsidering of the metaphors and images that are used in the pandemic era so as to understand the concerns about the language that is used to describe them and what effect such metaphors have on the general public.

There is no doubt however in the fact that metaphors have played a major part in spreading information about the pandemic and in sensing it's grave nature. The way in which it has created similarities between different forms of images with the pandemic related concerns is also an important element that grounds the relationship between pandemic and the English language.

\section{Emergence of Corona Poetry, Stories and Slogans}

The pandemic has put a break on everything and given people a great amount of time to reflect upon their life closely. It has made most of us write and produce a lot of literature about the pandemic experiences and to reflect upon the various areas of our life that are affected by it. For most of the time this reflection has come out in the form of poetry, stories and slogans. Undoubtedly, historical events have always powered the pen of many people to write and if we take a glance at history, major events such as the 17 th century plague, World War I, World War II and similar events have ignited many minds to write poetry, stories, novels and other forms of literature.

The new reality and lifestyle that the pandemic has offered us has been versified and textualized by people across the world. There are a variety of themes such as isolation, quarantine, sickness, loneliness, distancing, nature and relationships about which people have written largely since the pandemic has arrived. Sometimes, poetry has been used as a coping mechanism against the pandemic experience and at other times to give an outlet to the passions that were lost in the race of life that we all have been running in the pre-pandemic era.

Some of the corona literature that has been trending on various social media platforms include the poem "Lockdown" by Richard Hendrick, "A Song", "A Ballad" and "A Prayer" by Aditya Jadhav, "Kunjiamoomma" stories by Meena Shaktivanam and the poem "An Imagined Letter from COVID-19 to Humans" by Kristin Flyntz. The array of slogans that have appeared popular during the pandemic are also noteworthy and include the most widely used such as 'Get vaccinated before that first cuddle', 'If you get vaccinated, we will meet again; but if you neglect, we will meet in the grave', 'Vaccinate and stay alive', and 'Mask up'. Such slogans, poetry, and stories have enriched the English language as well as helped in circulating the coping mechanisms against the pandemic.

\section{Changes in the Language of Virtual Communication}

Never in history, virtual mode of communication has been used at such a massive level in every sphere of life before the arrival of COVID 19 pandemic. Since it's first blow, the virtual mode of communication has taken over the previously predominant personal mode. Virtual platforms of communication such as Zoom, GoogleMeet, Microsoft Teams and other tools of video-conferencing have been used worldwide to cope up with the need of the spheres where earlier face to face communication could not be substituted with anything else. Language has also been largely affected by this switch to virtual communication.

In face-to-face communication the language that was used and the time constraint was a major factor in the effectiveness of the process whereas on virtual platforms, the communication process has been made flexible to a greater extent. This has its own advantages and disadvantages but the language that is used in virtual modes of communication needs to be highly refined and appropriate to the situation because of the greater chances of 
miscommunication while on the other hand in person interaction has higher chances of solving these miscommunications and misunderstandings.

\section{Effects of Pandemic on the Teaching of English Language}

Worldwide travel restrictions, lockdown, and the closure of educational institutions have changed the whole course of imparting education at various levels. In this connection, the pandemic has changed the very core of the way in which the English language had been taught earlier and has posed new challanges for both the learners and the teachers. Distance learning and e-learning of English has taken over and replaced the traditional teaching methods due to the restrictions on personal interactions. However, the teaching of English as a language has been competitively adapting to the situation and many new online platforms for learning the English language have been effectively and extensively used during the time of crisis. But the pandemic has forced us to understand the challenges and make strategies to further develop e-learning platforms for the learning of English and cope up with the unpredictable situations that can also occur in the future.

\section{Conclusion}

The impact of COVID 19 pandemic on the education sector in general and English language in particular has given a brief overview of the loopholes and drawbacks as well as the strengths in the existing teaching-learning scenario of the English Language. It has also made us revaluate and reconsider the power of language which is the core of our human existence. The multidimensional use of English language during the time of pandemic has also reassured that the power of a global language should not be misrepresented. An understanding of the multiple imprints of the pandemic COVID 19 over the English language can further enlighten about the prospective areas that need to be explored in order to mitigate the upcoming crises and making the language readily capable of handling such situations with effectiveness and strength.

\section{Works Cited}

AI, Saman S, and Haider Ali. "COVID-19 Trending Neologisms and Word Formation Process in English". Russian Journal of Linguistics, vol. 25, no. 1, 2021, pp. 22-32, doi10.22363/2687-0088-2021-25-1-24-42.

Drucker J, Donna, and Karen Fleischhaver. "Language Pedagogy in a Pandemic: The Shift to Online Instruction During the COVID-19 Crisis". Journal of Pedagogical Research, vol. 5, no. 1, 2021, pp. 36-45, dx,doi.org/10.33902/JPR.2021167474.

Eraslan, Ali. "English Language Teaching and Learning During Covid-19: A Global Perspective on the First Year". Journal of Educational Technology and Online Learning, vol. 4, no. 2, 2021, doi.org/10.3168/jelol.907757.

Panzeri, Francesca, et.al. "Does the COVID-19 War Metaphor Influence Reasoning”. Plos One, vol. 16, no. 4, 2021, pp. 49-53, doi.org/10.1371/journal.pone.250651.

Putra, Adi Kristian, and Nur Arifah Drajati. Post Pandemic L2 Pedagogy. Routledge, 2021.

Semino, Elena. "Not Soldiers but Fire Fighters- Metaphors and Covid-19”. Health Communications, vol. 36, no. 1, 2021, pp. 90-96, doi.org/10.1080/10410236.2020.1844989. 